\title{
Ultra-high-speed all-channel serial-to-parallel conversion based on complete optical fourier transformation
}

\author{
Guan, Pengyu; Røge, Kasper Meldgaard; Morioka, Toshio; Oxenløwe, Leif Katsuo
}

Published in:

Proceedings of 2016 Optical Fiber Communications Conference and Exhibition

Link to article, DOI:

10.1364/OFC.2016.W3D.2

Publication date:

2016

Document Version

Peer reviewed version

Link back to DTU Orbit

Citation (APA):

Guan, P., Røge, K. M., Morioka, T., \& Oxenløwe, L. K. (2016). Ultra-high-speed all-channel serial-to-parallel conversion based on complete optical fourier transformation. In Proceedings of 2016 Optical Fiber Communications Conference and Exhibition (pp. 3 pp.). [W3D] Optical Society of America (OSA). https://doi.org/10.1364/OFC.2016.W3D.2

\section{General rights}

Copyright and moral rights for the publications made accessible in the public portal are retained by the authors and/or other copyright owners and it is a condition of accessing publications that users recognise and abide by the legal requirements associated with these rights.

- Users may download and print one copy of any publication from the public portal for the purpose of private study or research.

- You may not further distribute the material or use it for any profit-making activity or commercial gain

- You may freely distribute the URL identifying the publication in the public portal 


\title{
Ultra-high-speed All-channel Serial-to-parallel Conversion based on Complete Optical Fourier Transformation
}

\author{
P. Guan, K. M. Røge, T. Morioka, L. K. Oxenløwe \\ DTU Fotonik, Technical University of Denmark, Ørsteds Plads, 343, Kgs. Lyngby, 2800, Denmark \\ pengu@fotonik.dtu.dk
}

\begin{abstract}
We propose a serial-to-parallel conversion scheme based on complete OFT, allowing simultaneous conversion of all channels. We demonstrate all 32-channel simultaneous OTDM to WDM conversion of 320-Gbit/s DPSK and of 640-Gbit/s DQPSK signal, respectively. OCIS codes: (060.4510) Optical communications; (060.4230) Multiplexing; (070.7145) Ultrafast processing.
\end{abstract}

\section{Introduction}

Due to the rapid traffic growth in optical communication networks, intensive efforts have been made to develop new technologies for the future high capacity energy-efficient communication systems [1]. Presently, optical serial-toparallel conversion of a high-speed optical signal to several low-speed channels at different wavelengths, has received much attention. Since it allows high-speed complex serial signal processing to be achieved in parallel with low-speed or even passive components such as ultrafast optical oscilloscope [2], 640-Gbit/s clock recovery [3], and demultiplexing of optical time-division multiplexed (OTDM) data [4]. The serial-to-parallel conversion in these demonstrations were realized by time-lens based optical Fourier transformation (OFT) [5], which can transfer the temporal profile of an optical signal into the frequency domain and vice-versa. However, these demonstrations were achieved by a "partial" OFT, allowing only a part of the serial channels to be converted to parallel channels. An allchannel serial-to-parallel converter where all channels are simultaneously converted may become a very important functionality, since it may enable the simultaneous processing of a large number of parallel channels (e.g. WDM data) in a single device. This can be achieved by intermediately converting the parallel signal to a serial one and then applying high-speed optical serial signal processing techniques, followed by serial-to-parallel conversion. For example, WDM regeneration of differential phase-shift keying (DPSK) signals using a single phase sensitive amplifier has been demonstrated with this approach [6]. However, all-channel serial-to-parallel conversion is still very challenging, and it has only been demonstrated once using an improved "partial" OFT, although at the cost of a large power penalty due to inter-channel crosstalk [7].

In this paper, we propose an all-channel serial-to-parallel conversion scheme based on a "complete" OFT. Using this scheme, all 32 tributaries of a 32 x 10 Gbit/s DPSK-OTDM signal are simultaneously converted to 32 DWDM channels with $0.3 \mathrm{~nm}$ channel spacing. Full system characterizations with bit error rate (BER) measurements are performed, demonstrating a BER performance below $10^{-9}$ for all 32 converted channels. Furthermore, differential quadrature phase-shift keying (DQPSK) modulation is applied to demonstrate all-channel 640-Gbit/s OTDM to WDM conversion with a BER performance below the FEC limit $\left(2 \times 10^{-3}\right)$. To the best of our knowledge, these are the highest bit rate demonstrations of all-channel serial-to-parallel conversion.

\section{Principle}

The OFT is based on the principle of a "time-lens", originating in the space-time duality of light stating that quadratic phase modulation (a time-lens) of a temporal waveform is analogous to the action of a thin lens on a spatial beam [8]. Hence, by combining a time-lens with suitable dispersion both before and after the lens, an OFT can be realized as shown in Fig. 1(a), where two dispersive media with $D=\beta_{2} L\left(\beta_{2}\right.$ is the second order dispersion and $L$ is the length), separated by a quadratic phase-modulation stage $\left(\delta \phi=K t^{2} / 2\right)$ with chirp rate $K$, satisfies the condition $K=1 / D$ (a $D-K-D$ configuration). Although such an OFT has been used for serial-to-parallel conversion in many demonstrations, it is not suitable for all-channel conversion due to a fundamental limit of the $D-K-D$

(a) Traditional OFT (D-K-D configuration)

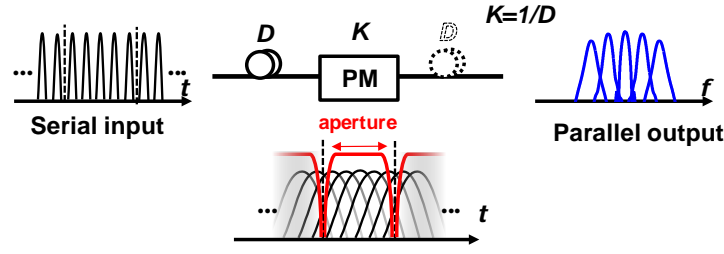

(b) Proposed complete OFT (K-D-K configuration)

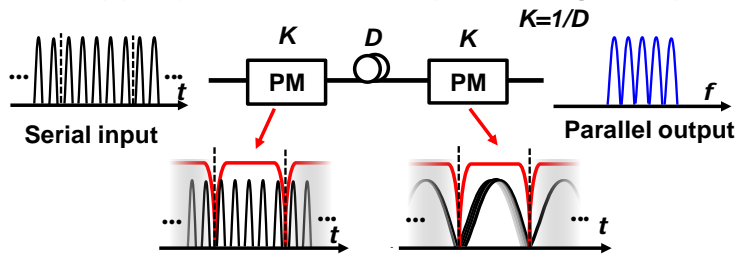

Fig. 1. Schematic diagrams of a time-lens based OFT. (a) traditional D-K-D configuration, (b) proposed K-D-K configuration for all-channel serial-to-parallel conversion. 


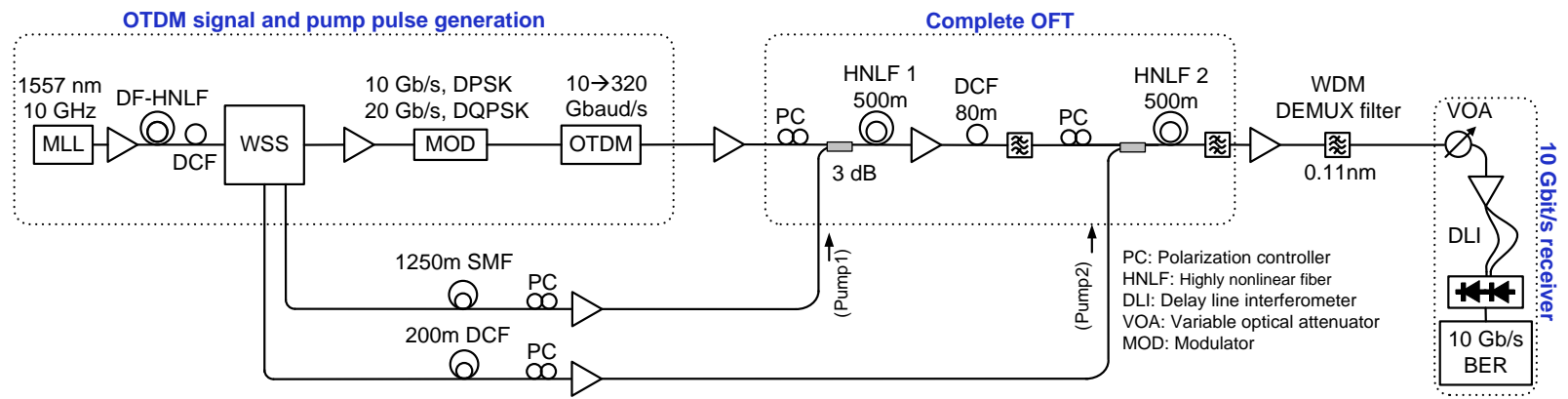

Fig. 2. Experimental setup for all-channel OTDM to WDM conversion of a 320-Gbit/s-DPSK signal or a 640-Gbit/s-DQPSK signal.

configuration as illustrated in Fig. 1(a). The dispersive elements involved before the phase modulation stage will generally broaden the input waveform, making the edge serial tributaries partially extend beyond the temporal aperture of the time-lens. This will cause temporal clipping and hence spectral broadening and power loss of the edge channels after serial-to-parallel conversion, resulting in a large inter-channel crosstalk and OSNR degradation. To overcome this challenge, we propose to use a new time-lens based complete OFT, which has also been used in demonstrations such as WDM to Nyquist-OTDM conversion [9] and OFDM to Nyquist-WDM conversion [10]. The schematic diagram of this OFT is shown in Fig. 1(b), where two quadratic phase-modulation stages with chirp rate $K$, separated by a dispersive medium with $D$, which satisfies the condition $K=1 / D$ (a $K-D-K$ configuration). With this configuration, all input serial tributaries are pre-chirped by the first time-lens. When the chirped tributaries pass though the dispersive medium, they will not only experience the waveform broadening, but also become aligned (focused) to the center of the second time-lens aperture. This configuration can thus keep the waveform within the time-lens apertures, making it suitable for a simultaneous, all-channel serial-to-parallel conversion (a "complete" OFT). The chirp rate $K$ determines the scaling factor between the time- and frequency- domains according to $\Delta t=2 \pi \Delta f / K$.

\section{Experimental setup and results}

In the experimental demonstration, a high-speed 320-Gbaud serial data signal is generated by OTDM. The experimental setup is shown in Fig. 2. A $10 \mathrm{GHz}$ mode-locked laser (MLL) is used as an optical pulse source, generating a $1.5 \mathrm{ps}$ pulse train at $1557 \mathrm{~nm}$. This is amplified and spectrally broadened by self-phase modulation in a $400 \mathrm{~m}$ dispersion-flattened highly non-linear fiber (DF-HNLF). The broadened spectrum is Gaussian-filtered at $1546 \mathrm{~nm}$ by a programmable wavelength selective switch (WSS). The obtained 1 ps pulse train is DPSK or DQPSK modulated using an IQ modulator. The 10-Gbaud modulated signal is then OTDM-multiplexed to 320-Gbaud using an OTDM multiplexer, resulting in a 320-Gbit/s DPSK-OTDM signal or a 640-Gbit/s DQPSK-OTDM signal. Fig. 3(a) shows the obtained 320-Gbaud OTDM signal waveform with a 3.02 ps tributary spacing. A 3.4 ps guard interval is inserted between every 32 tributaries to allow for the transition between consecutive quadratic phasemodulation windows.

A complete OFT is used for the all-channel serial-to-parallel (OTDM-to-DWDM) conversion. The quadratic phase modulation is implemented based on a four-wave mixing (FWM) process in a highly nonlinear fiber (HNLF) using linearly chirped (quadratic phase) rectangular pump pulses at $10 \mathrm{GHz}$. Both pump pulses and the data signal are obtained from the same broadened spectrum using a WSS. To obtain linearly chirped pumps, the broad spectrum is filtered using the WSS, and each pump is subsequently propagated in an appropriate length of dispersive fiber. Pump $_{1}$ is dispersed in a $1250 \mathrm{~m} \mathrm{SMF}$ to achieve a chirp rate $K=0.078 \mathrm{ps}^{-2}$ for conversion of the $3.02 \mathrm{ps}$ temporal spacing to a $37.5 \mathrm{GHz}(0.3 \mathrm{~nm})$ frequency grid. As the data-signal is phase-conjugated after the first FWM process,

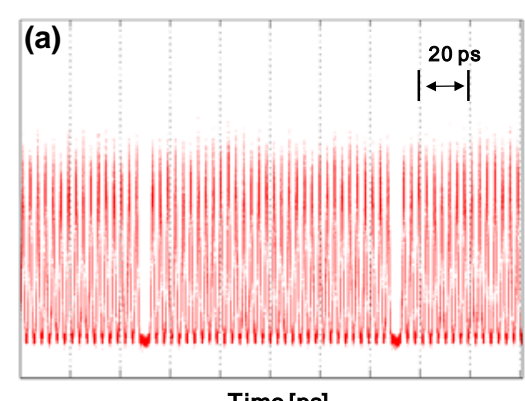

Time [ps]
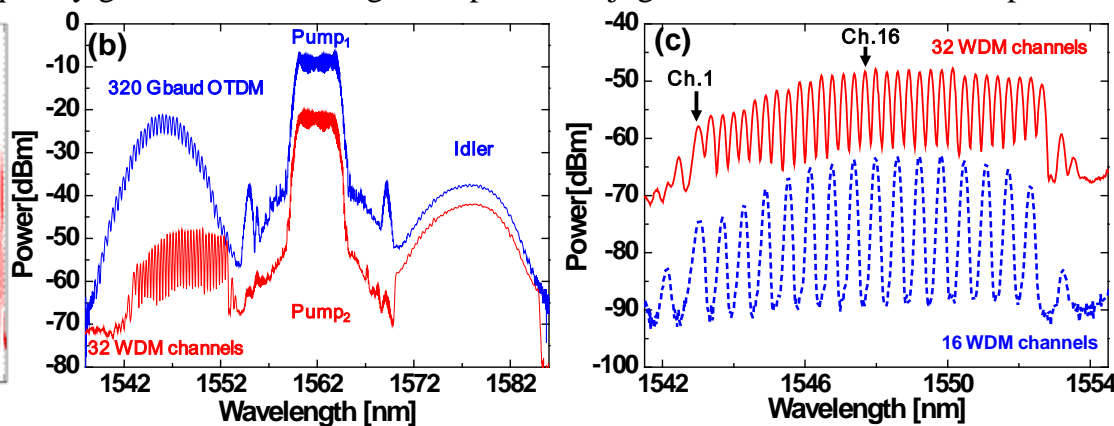

Fig. 3. Results of OTDM to WDM conversion. (a) 320 Gbaud OTDM signal waveform, (b) optical spectrum after the first (blue) and second (red) FWM process, (c) obtained 32 WDM channels (red) and 16 WDM channels obtained by blocking the adjacent OTDM tributaries (blue). 

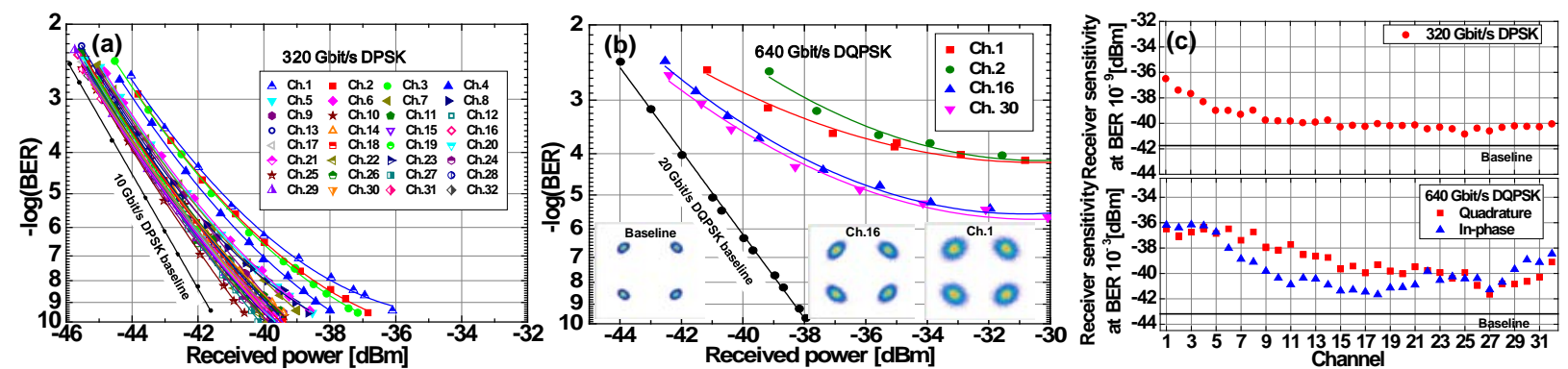

Fig. 4. Experimental result. (a) BER performance of all 32 converted 10-Gbit/s DPSK-WDM channels, (b) BER performance for some converted DQPSK-WDM channels, (c) receiver sensitivities at BER $=10^{-9}$ of all converted DPSK-WDM channels, and receiver sensitivities at $\mathrm{BER}=10^{-3}$ of all DQPSK-WDM channels.

pump $_{2}$ is dispersed in $200 \mathrm{~m}$ of dispersion compensating fiber (DCF) having the opposite dispersion value of the $1250 \mathrm{~m} \mathrm{SMF}$, in order to achieve the same chirp rate $K$ in the second time-lens. The central wavelengths of the pumps are set to $1562 \mathrm{~nm}$. The first FWM output is shown in Fig. 3 (b). After extraction with a $15 \mathrm{~nm}$ optical bandpass filter (OBPF), the idler is dispersed in an $80 \mathrm{~m}$ DCF corresponding to dispersion D, and subsequently combined with pump 2 and coupled into $\mathrm{HNLF}_{2}$ for the second FWM process. The resulting spectrum is also shown in Fig. 3(b). The generated idler is the 32-channel WDM signal converted from the 320-Gbaud OTDM signal. Fig. 3(c) shows a zoom-in on the generated idler, where $32 \mathrm{WDM}$ channels with $37.5 \mathrm{GHz}(0.3 \mathrm{~nm})$ spacing can be observed. When every second OTDM tributary is blocked (resulting in 16 WDM channels with 75 GHz spacing), a high extinction ratio (ER) can be observed, where $25 \mathrm{~dB}$ and $17 \mathrm{~dB}$ are for the center and left edge channels. The skew between the channels is caused by the limited FWM bandwidth of the used HNLFs. This indicates a nearly insignificant spectral overlapping, resulting in a crosstalk-less all-channel serial-to-parallel conversion. In the receiver, a $14 \mathrm{GHz}(0.11 \mathrm{~nm})$ Gaussian filter is used to demultiplex each WDM channel. Finally, the BER of each demultiplexed DPSK or DQPSK channel is measured in a pre-amplified receiver including a delay-line interferometer (DLI) and a balanced photo-detector.

Fig. 4(a) shows the BER measurements for all 32 converted 10 Gbit/s DPSK-WDM channels, where error-free operation $\left(\mathrm{BER}<10^{-9}\right)$ is achieved for all WDM channels with an average receiver sensitivity of $-39.7 \mathrm{dBm}$. The average power penalty at $\mathrm{BER}=10^{-9}$ is $2 \mathrm{~dB}$ compared to the $10 \mathrm{Gbit} / \mathrm{s}$ DPSK baseline. Fig. 4(b) shows the BER measurements for some selected DQPSK-WDM channels converted from the 640-Gbit/s DQPSK-OTDM signals. As the DQPSK signals have a higher OSNR requirement and a lower phase noise tolerance than DPSK signals at the same baud rate, there are error floors around $10^{-5}$ and $10^{-6}$ due to the nonlinear phase noise and OSNR degradation introduced by the FWM processes. The constellations for Ch.1, Ch.16 and the DQPSK baseline obtained using a coherent receiver with maximum received power are also shown in the inset, in which nonlinear phase noise and OSNR degradation can be observed after conversion. Fig. 4(c) shows the corresponding receiver sensitivities at $\mathrm{BER}=10^{-9}$ of all converted DPSK-WDM channels, as well as the receiver sensitivities at BER $=10^{-3}$ of all DQPSKWDM channels. It can be seen that the BER performance of the left edge channels are worse than the other channels. This is mainly due to the OSNR degradation caused by the limited FWM bandwidth of the HNLFs, which can also be confirmed in Fig. 3(c). The DQPSK demonstration confirms the fact that the proposed scheme preserves the phase information, and can be applied to complex modulation formats.

\section{Conclusion}

We have proposed an ultra-high-speed all-channel serial-to-parallel conversion scheme based on complete OFT. All 32 tributaries of a 320-Gbit/s DPSK-OTDM signal were simultaneously converted to 32 DWDM channels, achieving a BER $<10^{-9}$ performance with only $2 \mathrm{~dB}$ average power penalty. By using DQPSK modulation, an allchannel 640-Gbit/s DQPSK-OTDM to WDM conversion was also demonstrated with a BER performance below the standard FEC threshold $\left(2 \times 10^{-3}\right)$. This scheme has the potential to extend the existing serial signal processing techniques to include parallel processing, which may improve the energy-efficiency for future communication systems.

Acknowledgements: The authors would like to thank OFS Denmark for supplying the nonlinear fibers. This work is supported by the FTP-TOR project (ref. no. 12-127224) and the DFF Sap. Aude Adv. Grant NANO-SPECs.

\section{References}

[1] L. K. Oxenløwe et al., IEEE JSTQE.18, 996-1005, (2012).

[2] M. Foster et al., Nature 456, 81-84, (2008).

[3] H. C. H. Mulvad et al., Proc. ECOC, Mo.1.A.6, (2010).

[4] H. C. H. Mulvad et al., Proc. OFC, OThN2, (2011).

[5] M. Nakazawa et al., IEEE PTL. 16, 1059-1061, (2004).
[6] P. Guan et al., Proc. ECOC, We.3.6.4, (2015)

[7] K. G. Petrillo et al., Proc. CISS, 1-6, (2013).

[8] B. H. Kolner et al., Opt. Lett. 14, 630- 632, (1989).

[9] P. Guan et al., Proc. ECOC, We.2.5.5, (2014).

[10] P. Guan et al., Proc. OFC, W3C.6, (2015). 